\title{
ONLINE MEDIATION: A GAME CHANGER OR MUCH ADO ABOUT NOTHING?
}

\author{
Victor Terekhov \\ PhD in Law, lecturer of European Civil Procedure \\ and European Private Law, \\ Vilnius University (Vilnius, Lithuania)
}

doi.org/10.33327/AJEE-18-2.4-a000018

\begin{abstract}
Summary: 1. Introduction - 2. Defining Online Mediation. - 3. Technologies Used in Online Mediation. - 4. Areas of Application. - 5. Known Benefits. - 6. (Un)obvious Drawbacks. - 7. Solutions and Potential for Improvement. - 8. Concluding Remarks.
\end{abstract}

This paper focuses on the phenomenon of Online Mediation, which is gaining in popularity in recent years. Being part of the Online Dispute Resolution family, this particular method is the one applied most often. The very idea of disputes being heard and resolved in the global network seems exciting and quite appealing to some, while for others it presents a source of major concern. New technologies influence the ways parties and the neutral interact, share ideas and reach a settlement. Moreover, they have a clear impact on how people evaluate the other party, their mediator and the whole procedure they are involved into. This makes trust a significant issue for online mediation, one that is not so easy to establish while relying on the old techniques. Another important thing is the absence of positive regulation for the sector. In spite of recent instruments adopted by the EU, online mediation is still a field largely unknown to lawyers, consumers, business players and national regulators.

The present article aims at clarifying the notion of 'online mediation' (which, surprisingly, has not been properly done yet), showing some of the most obvious benefits and drawbacks of this dispute resolution method (a deeper, more profound look on them will only be possible over time, when online mediation proves itself in practice and more statistical data are available) and providing valuable remarks on the solutions for the problems determined.

Key words: Online Dispute Resolution, Online Mediation, IT platforms, fourth party, consumer disputes, eCommerce, caucusing, settlement of disputes

\section{INTRODUCTION}

In the past decade we have witnessed a rapid entry of technologies into our lives. Businesses, consumers and governments start to perform their usual tasks with the help of computers, and many new previously unknown fields of human interaction come to 
life. Being formerly an object of interest exclusively to software engineers and a small IT community, now the technology is a powerful tool changing the world on a daily basis. ${ }^{1}$ More and more people choose a career in the IT sector, while those, whose work never involved any interaction with computers, are now intensively trying to catch up with the modern technological trends.

It is hard to imagine an area that has not recently moved online: one can name banking, insurance, consulting, education, commerce, consumer shopping, entertainment and even certain public services provided by governments and municipalities. ${ }^{2}$ At the same time, many new sectors and industries are emerging, such as trading in domain names, web hosting, online gaming, cloud storage of data, ${ }^{3}$ blockchain and cryptocurrencies, smart contracts ${ }^{4}$ with more to come in the foreseeable future.

While appreciating this remarkable progress, we must not forget about the problems that go in hand with it. People still face difficulties entering into relations with each other: numerous disputes arise and put obstacles on further development and bring too much of a fuss to all involved. Luckily, humanity has already developed civilized ways of dispute resolution helping overcome disagreements and restore peace and harmony. These may be adjudicatory or non-adjudicatory, binding or non-binding, mandatory or voluntary, facilitative or evaluative, involving a third neutral party or just the two disputants. All of them nowadays follow the general trend and also relocate to the web. There are several reasons for this: convenience for internet-people, ${ }^{5}$ lower costs, simplicity and speed of procedures, and, last but not least, the opportunity for providers of related services to compete for yet another important market (that of internet dispute resolution). ${ }^{6}$

This article focuses on one particular method of dispute resolution, namely, online mediation, as it seems to be one of the easiest to be deployed online (unlike, e.g. online arbitration facing difficulties with recognition, establishing a proper form for agreement and the award and some other). Mediation is also among the most popular ways of dispute resolution in real ('offline') life, ${ }^{7}$ thus there is no reason things should dramatically change when relocating online. ${ }^{8}$

Part II of this article elaborates on the definition of Online Mediation, describing it as an Online Dispute Resolution (ODR) technique, and sheds some light on the history of the matter. Part III presents an overview of the technology used to conduct mediation sessions and to help parties and their mediator reach a peaceful settlement of the dispute. In Part IV an incomplete list of areas where Online Mediation is already widespread, or may become popular in the future, is provided. Parts V and VI describe known

$1 \quad$ P Astromskis, 'Ateities teisès tyrimų modelis' (2018) 1(17) Teisès apžvalga 74.

2 O Turel, Y Yuan, J Rose, 'Antecedents of Attitude towards Online Mediation' (2007) 16(6) Group Decision \& Negotiation 539.

3 M Corrales, M Fenwick, N Forgó, Disruptive Technologies Shaping the Law of the Future in: New Technology, Big Data and the Law (Springer 2017) 2.

4 R O'Shields, 'Smart Contracts: Legal Agreements for the Blockchain' (2017) 21 North Carolina Banking Institute 179.

5 'Internet people' (an 'internet person' for a particular representative) - people who live on the Internet, follow all of its trends and feel more comfortable in the online environment than they do in real life. See the definition in Urban Dictionary <https://www.urbandictionary.com>.

6 J Hörnle, Cross-border Internet Dispute Resolution (Cambridge University Press 2009) 87.

$7 \quad$ P Cortés, 'Can I Afford Not To Mediate? Mandatory Online Mediation for European Consumers: Legal Constraints and Policy Issues' (2008) 35 Rutgers Computer and Technology Law Journal 1.

8 As shown in the research of Schultz, Kaufmann-Kohler et al, Online Mediation is indeed the most common form of ODR with over 50 institutions providing it. See T Schultz, G Kaufmann-Kohler et al, Online Dispute Resolution: The State of the Art and the Issues (University of Geneva 2001) 24. 
advantages and drawbacks of using this method, while Part VII shows particular ways of overcoming major hurdles. Part VIII concludes and presents some food and thought.

\section{DEFINING ONLINE MEDIATION}

Before trying to evaluate the phenomenon of Online Mediation it makes sense to find a proper definition for it. The concept itself is not new, appearing towards the end of the 1990s with the expansion of the Internet for commercial use. ${ }^{9}$

The most common definition of mediation as such is a method of ADR where an impartial person (mediator) assists parties in reaching an independent solution of their dispute. In the European Union mediation (at least for cross-border instances) is defined as a 'structured process, however named or referred to, whereby two or more parties to a dispute attempt by themselves, on a voluntary basis, to reach an agreement on the settlement of their dispute with the assistance of a mediator.'10 National member states have their own regulations on mediation 11 and even special names for it and those practicing in this area. ${ }^{12}$

Mediation is commonly based upon the following rules and principles:

(1) It involves three parties - two disputants and a neutral intermediate offering his assistance. Although in principle there may be more conflicting subjects, in practice we normally have just two of them with the most opposite views on certain essential questions. The presence of the neutral is what distinguishes the process from negotiation where the parties communicate directly. Mediation is 'assisted negotiation' which is launched where the latter process fails. ${ }^{13}$ All mutual relations between the two parties and parties with their mediator are normally referred to as the 'mediation triangle'.

(2) Non-adjudicatory role of the mediator. The third neutral party has no adjudicatory powers. His role is to facilitate the conversation between the main parties by providing them with advice, opinions, suggestions, directions and information. In some variations of the procedure he may even present a draft settlement, but to sign it and impose its contents upon the parties is definitely beyond his authority. Such a prerogative is reserved for the disputants. In most situations the goal of the mediator is to create a settlement-friendly atmosphere, whatever that can mean in a particular case. It must be noted that the third party must be independent from the disputing parties and impartial in his views and appreciation of the dispute.

(3) Flexible and informal nature of the procedure. Mediation is only regulated by the law in most abstract terms. The fundamental parts of the procedure are decided by the three parties or are laid down in terms and conditions of the mediator. Flexibility means fewer

9 Previously, in the early 1990s the Internet was mainly used by scholars, educational and military institutions, while the conflicts within it were quite rare and, in any case, preference was given to judicial ways of dispute resolution.

10 Directive 2008/52/EC of the European Parliament and of the Council of 21 May 2008 on certain aspects of mediation in civil and commercial matters (EU Mediation Directive), OJ L 136, 24.05.2008, 3-8.

11 E.g. In the Republic of Lithuania, mediation is understood as 'a dispute resolution procedure in which one or more mediators help the parties to the dispute to resolve the dispute amicably'. In most cases it is seen as a professional activity (Law on Mediation, 15.07.2008, Nr. X-1702, Art. 2).

12 Thus, in Estonia the law distinguishes between 'mediation' and 'conciliation', in Greece besides ordinary mediation there are similar processes (with a different status) for employment and consumer cases, etc. See: Mediation in EU Member States information page - <https://e-justice.europa.eu/content_ mediation_in_member_states-64-be-en.do?member=1> accessed 15 August 2019.

13 E M Lombardi, 'Is Online Mediation the Way to Fit the Forum to the Fuss?' (2012) 19(4) Maastricht Journal of European and Comparative Law 526. 
bureaucratic formalities and obligatory steps to be satisfied in order for the process to gain recognition. It also means that parties are in the control of the procedure and can construct it in accordance with their wishes. When we talk about an informal style of mediation we mean that it is an 'interest based procedure' in contrast with litigation or arbitration ('right-based procedures'). 14 The aim here is to determine what the parties can do for each other rather than to establish who is right and wrong.

(4) Private origins. On the one hand, mediation is mainly used to deal with disputes of private (i.e. 'non-public' nature), those that parties are allowed to dispose of themselves, without governmental intervention. This feature is slowly becoming obsolete, since we find a growing number of mediation examples in criminal, administrative, constitutional and even international law. On the other hand, the most important thing is that mediation is being offered by professionals who are not part of the judiciary or other governmental authority. In majority of cases these persons only receive certificates or training from a public authority, being virtually independent and not subject to any review on matters of law. In some states mediators are a self-regulated profession while in a small number of them it is not regulated at all.

(5) Voluntary participation. Parties may not be forced to mediate as any resort to this procedure is a matter of mutual and independent agreement. However, in modern doctrine of civil procedure it is believed that settlements are generally preferable to 'hard' solutions, thus some national legislators design a system of mandatory, or court imposed mediation.15 At the same time, even such procedures do not fully block subsequent access to judicial procedures (otherwise would be a violation of the ECHR Art. 6 on fair trial or the national provisions with a similar effect). Voluntary nature means autonomy for the parties to participate and/or leave the process at any time desired. They will not be punished for their lack of cooperation or inability to arrange a settlement. The content of the final document (mediation agreement) is also up to the parties, although the mediator may propose some template to follow.

(6) Mostly face-to-face interaction. Although not included in the notion of mediation (supposedly on purpose), mediation is normally a face-to-face process, such where the parties see each other (being virtually present together in mediator's cabinet) and may engage in oral discussions. This is regarded as essential, since otherwise it is extremely hard to build up a settlement-friendly atmosphere.

In the end, mediation presents an effective way of dispute resolution, since it helps operatively solve the problems and alleviate the pressure on courts. ${ }^{16}$ What about online mediation then? How is it related to classic mediation and what is so special about it? First of all, it is usually seen as an integral part of Online Dispute Resolution (ODR) movement, which to most speakers presents the use of technology to assist parties with the resolution of a dispute outside the courtroom. ${ }^{17}$ The exact meaning and contents of the term ODR is, however, subject to controversy. Most scholars relate ODR with ADR, which makes sense as the most common forms of the former (apart from mediation) are negotiation and arbitration - which are also the most well-known types of ADR. ${ }^{18}$ In this way, online dispute resolution stands

\footnotetext{
$14 \quad$ Cortés (n 7) 1.

15 See N Kaminskienè, 'Privaloma mediacija: galimybès ir iššūkiai' (2013) 20(2) Jurisprudencija 687-8.

16 A V Feoktistov, 'Mediation as a Means of Conflict Regulation' (“Mediatsiia kak sposob uregulirovaniia konfliktov') (2014) 27 Concept 1.

17 J C Betancourt, E Zlatanska, 'Online Dispute Resolution (ODR): What Is It, and Is It the Way Forward?' (2013) 79 Arbitration 256.

18 K Mania, 'Online Dispute Resolution: The Future of Justice' (2015) 1 International Comparative Jurisprudence 78 .
} 
for nothing else than traditional ADR supplemented and facilitated by some modern information and communication tools (ICT), such as email, VoIP, smart-messengers, videoconferencing and so on.

At the same time, many scholars tend to give ODR a more independent status. ${ }^{19}$ According to Hörnle, ODR is not just a transplant of ADR into the online environment, as we need never underestimate the transformative power of the technology. ${ }^{20}$ In online forms of dispute resolution the latter is not only used as communication medium, but takes the active role, assisting the third party in resolving the dispute and on rare occasions even taking its role. ${ }^{21}$ The pioneers of the ODR movement, E. Katsh and J. Rifkin even coined the term of 'fourth party' to describe a special status of the technology. ${ }^{22}$ Computer tools help organize sessions, collect and sort data, present relevant materials to the parties at a convenient time, remind of the sessions and deadlines to submit documents, analyze previous practice and on that basis propose solutions for the case at hand. In other words, programs can do much more than a human individual, consequently they are not being used, but are rather functioning on their own, performing their separate and unique tasks. Another view insists on drawing a line between popular technologies (such as email or Skype) created and used for different tasks and sectoral technologies, which are more sophisticated and normally designed precisely for dispute resolution purposes. Only applying the latter ones we deal with ODR, while the use of traditional web instruments does not create any added value, and consequently does not need additional attention and research (it is not ODR in its pure form, but rather technicallyfacilitated dispute resolution).

Applying everything mentioned to online mediation, we can note that in many aspects it mirrors its traditional offline counterpart. ${ }^{23}$ It also takes place between two parties and an intermediary, the latter not having right to impose a decision, and it also concentrates on interests instead of rights. The difference lies in the active use of information and communication technology (ICT). Consequently, for many of us online mediation is just the same process empowered and supported by modern technology. ${ }^{24}$ The only difference seemingly lies in the device used to connect the participants.

As was just shown, this is quite a simplistic idea of what the whole process is. Technologically advanced mediation is not the same thing as online mediation. Despite being quite close to each other, they have sufficient differences, which may become evident through an example. Imagine a mediator knowing his client to be away from his normal business premises, decides to organize a videoconference between the parties or to keep in touch through email. This does not necessarily turn the whole process into an 'online' one. ${ }^{25}$ As was said, computer technologies are so much a part of our daily lives that their use for both personal and professional issues does not present further

19 L Zissis, 'Disputes in the Digital Era: The Evolution of Dispute Resolution and the Model ODR System. (Université de Toulouse 2015) 153.

20 J Hörnle (n 6) 86.

21 G Ross, 'ODR's Role in In-Person Mediation and Other 'Must Know' Takeaways About ODR' <https:// www.mediate.com/articles/RossG2.cfm> accessed 10 August 2019.

22 E Katsh, J Rifkin, Online Dispute Resolution: Resolving Conflicts in Cyberspace (Jossey-Bass, 2001) 9.

23 A M Braeutigam, 'Fusses that Fit Online: Online Mediation in Non-Commercial Contexts' (2006) 5(2) Appalachian Journal of Law 285.

24 A Ramasastry, 'Government-to-Citizen Online Dispute Resolution: A Preliminary Inquiry’ (2004) 79 Washington Law Review 160.

25 Such a situation though is described as 'hybrid mediation' when the mediator can choose which solutions to rely upon. See Rogers, 365. 
questions, nor rise any specific problems to be solved. ${ }^{26}$ Moreover, we clearly see that in the situation given the use of distant communication means was only one of the available options. A mediator could alternatively postpone the session to a later date and prepare for a normal oral hearing. This shows that the use of technology in this and similar situations is only ancillary, there is no strict dependence on it and it is easily interchangeable. ${ }^{27}$

Quite the opposite will be the situation where the mediation takes place entirely online. On that occasion the participants use only one means of communication, do not get together physically and only interact online. The role of the mediator remains the same then, but the selection of techniques will sufficiently differ. ${ }^{28}$ No longer will it be possible to rely on facial expressions, gestures and body language of the clients. The atmosphere of interaction, its pace, emotions of the participants, worries and fears they encounter - everything will be slightly different. At the same time, technology will also permit some types of relations impossible in real life (e.g. immediate web sessions in chat rooms between each of the parties and mediator - previously he had to conduct private caucuses ${ }^{29}$ at a different time).

In extreme situations we may even replace a human-mediator with pre-programmed algorithms that respond to parties' behavior and actions and help them draft a solution. The necessary technologies are already here, and the reasons for their inactivity are rather legal and bureaucratic than technological. The programs will need some time, though, to take into account all peculiarities of human behavior and the spectrum of reactions to the same impulses. Moreover, people feel more comfortable working with other people, albeit indirectly, rather than depersonalized machines.

To sum up, we need to distinguish situations where classic procedures have embraced certain modern technologies from those where the IT tools present the driving force of the whole process and cannot be dispensed with. Only the latter ones will be further discussed here as 'online mediation', while for the former the term 'traditional mediation' will be used.

Online mediation started to be offered in the late 1990s and presented a purely university project then. Quite soon most projects evolved into commercial ventures and started to order professional internet services. ${ }^{30}$ However, most of them went bankrupt with the new millennium. The same was also true for academic research: while we find a vast amount of publications on the topic at the beginning of the 2000s, quite soon it ceased to be interesting and was largely abandoned. In recent years, however, we see a revival of interest for it. We can identify three reasons to this: (1) emergence of improved web technologies permitting to easily build up complex internet services for various use; ${ }^{31}(2)$ extreme workload of

26 H Pakaslanti, 'The Costs of Resolving Conflicts Online' (2017) < https://helda.helsinki.fi/ bitstream/handle/10138/191368/the\%20Costs\%20of\%20Resolving\%20Conflicts\%20Online. pdf? sequence $=2 \&$ is Allowed $=\mathrm{y}>$ accessed 29 July 2019.

27 G Kaufmann-Kohler, 'Online Dispute Resolution and Its Significance for International Commercial Arbitration' (2005) Commerce and Dispute Resolution 454.

28 P Cortés (n 7) 3-4.

29 Private, or separate caucus - 'a confidential mediation session that a mediator holds with an individual party to elicit settlement offers and demands'. See Black's Law Dictionary ( $9^{\text {th }}$ edition), ed. B A Garner (West 2009) 248.

30 J Hörnle (n 6) 75

31 There is a concept of 'software as a service' (SaaS), where a program designed by software engineers is deployed on a website and does not require installation and support on user's machine. On the other hand, it normally requires advance payments before an access to it can be obtained. Thus, it presents a perfect business model and one does only need to find a suitable niche to operate in. Dispute resolution virtually presents one of such 'prospective fields'. 
the courts, such that the government itself starts to look at ADR (and, now also ODR) as a saving grace; (3) relocation of most businesses and consumers to the web, where it is now comfortable and common to make transactions. As a consequence we see a unity of opinion concerning the necessity and importance of ODR.

Online mediation is still the most popular type of ODR and is being offered by a number of institutions (BBBOnline, Camera Arbitrale di Milano, Modria (Tyler), SmartSettle, SquareTrade, Web Trader, WebAssured, WebMediate and Internet Neutral). Despite that, this dispute resolution method, in the words of Cortés, is still in its infancy. ${ }^{32}$ Many issues surrounding it are problematic, the scholar and practicing community are not united in terms, no solid regulatory framework is provided from the public side and, finally, some potential users may be distracted from it due to trust-related problems.

\section{TECHNOLOGIES USED IN ONLINE MEDIATION}

Practice shows that Online Mediation is capable of using all range of available web-based technologies: from simple email communication and messengers to videoconferencing and procedures involving advanced algorithms. ${ }^{33}$ Despite the great diversity of solutions available on the market, they all may be divided into textual and dynamic (audio, video), and also immediate (synchronous) and asynchronous.

Textual, as the word suggests, rely on written statements submitted by the parties. Computers, tablets and mobile phones give the opportunity to exchange postings in various ways: SMS, instant messengers (IMs), web chats, forums (bulletin boards), social media sites, emails and others. Some of them may even be synchronous, e.g. permitting a person to see what the other one is typing, but in most cases it is only possible to reply after he/she has finished the message and sends it to you.

Dynamic tools include audio (phone calls, VoIP) and video (skype or another similar program) interactions. In most cases we are talking about their combination audiovisual mediation. ${ }^{34}$ Here parties can see each other and exchange their comments directly. Although in practice there is an example where a party 'films' a video, sends it to another party and then waits for a similar reply, ${ }^{35}$ there is more sense in a process where people are capable of seeing each other directly and engage in conversation in real time.

It must also be added that audiovisual mediation is an attempt to copy and even replicate the classic procedure of face-to-face communication: it is believed that such way of interaction gives a richer perspective on the case and permits the parties to be more open towards their companions and the intermediary. ${ }^{36}$ Since video-communication is, in principle, supported by any modern laptop or telephone, the proponents of online mediation normally speak out in favour of this type of procedure.

$32 \quad$ P. Cortés (n 7) 2.

33 E M Lombardi (n 13) 533-7.

34 F S Rossi, A Holtzworth-Munroe, 'Shuttle and Online Mediation: A Review of Available Research and Implications for Separating Couples Reporting Intimate Partner Violence or Abuse’ (2017) 55(3) Family Court Review 395.

35 See the example of Crowdsourced ODR: J van der Henrik, D Dimov, 'Towards Crowdsourced Online Dispute Resolution' (2011) Law Across Nations: Governance, Policy \& Statutes 244-257.

36 D Lavi, 'Till Death Do Us Part?!: Online Mediation as an Answer to Divorce Cases Involving Violence' (2015) 16(2) North Carolina Journal of Law and Technology 300. 
In spite of such ideas, online mediation is currently proposed principally in a written form. ${ }^{37}$ The reason is that broadband internet access is not evenly accessible throughout the world, making interactions between the parties from distant geographic regions via this method unavailable or significantly impeded. It may also create an unnecessary advantage for one of the parties, possessing better technical capabilities (normally big corporations vs. small and medium sized companies and consumers), while text-based instruments are widely distributed and universally available.

Among text-based solutions, email is the simplest way to conduct sessions between the disputants and the mediator. ${ }^{38}$ It is preinstalled in every computer, available as a web-service and requires no additional licenses or periodic payments. It is also rather fast and easy to use. Although popular in practice and heavily relied on, it is not among the best things the Internet can offer for disputing parties. This type of communication is not encrypted and thus prone to hacker attacks and the leakage of data. Moreover, its use does not give a mediator much power over the process. He is effectively prevented from using restrictive tactics and cannot basically influence the flow of postings between the parties. It is believed he might spend more time ensuring his authority than diving into the complex relations between the parties and the obstacles to their reconciliation. ${ }^{39}$

Much more effective are full-fledged online platforms specifically designed for dispute resolution purposes. Among their indisputable advantages one may name process-guided interfaces, smart reminders, virtual chat rooms, calendars, notes, drafts and much more. From among those, virtual meeting rooms present a particular interest and a decent alternative to email. These present a special interactive web-site hosted by the mediator (or, otherwise under his exclusive control) where parties may exchange notes and messages, and ultimately resolve their disagreements. They look like modern messengers enabling communicating subjects to see all the history of their correspondence. Virtual rooms may be general (open to all three participants) and private (only one of the parties and the mediator have access there). Such design enables the mediator to chat with both parties in separate browser windows and none of the parties is capable of seeing what is being typed to its counterpart. This seems impossible in real life, where the mediator has to conduct separate caucusing with both parties consequentially, which certainly increases the degree of tension (with each of the parties presuming something unfair happening behind the closed doors). ${ }^{40}$

Another solution worth mentioning is the dynamic filling forms. ${ }^{41}$ This is not artificial intelligence (AI) as such - we rather speak about pre-programmed web pages with data that appear in a particular order depending on which answers the party has chosen during previous steps. The forms change depending on the information entered. This solution, however, has its drawback: it does not allow much creativity, thus, instead of fully expressing herself (which is essential in a process like mediation) a party has to rely on answers and wording provided by the program. It must also be mentioned that computer algorithms are not yet ready to replace a human mediator, who is still a key player in the whole process. His ability to react quickly, change the tone and pace of

37 J Melamed, 'Mediating on the Internet: Today and Tomorrow' <https://www.mediate.com/articles/ Melamed5.cfm> accessed 20 July 2019.

38 F S Rossi, A Holtzworth-Munroe (n 34) 439.

39 R Regazzoni, 'RisolviOnline: Online Mediation from a Very Practical Point of View'<http://ceur-ws. org/Vol-430/Paper5.pdf> accessed 10 July 2019.

40 J Hörnle (n 6) 79.

41 Id 80. 
mediation, ask the correct questions and make reasonable comments is invaluable, and is not something a machine is likely to reproduce quite soon. ${ }^{42}$

Putting it together, technology in online mediation should provide for effective communication between the mediator and parties, establish a settlement-friendly atmosphere and present information and support required by the participants.

\section{AREAS OF APPLICATION}

The first type of disputes suitable and even preferable for online mediation that comes to our mind, is, probably, conflicts in eCommerce. The latter word comprises different forms of commercial activity in the Internet, including sales and purchases, provision of services and some other things. ${ }^{43}$ It may include both B2B and B2C transactions. ${ }^{44}$

ODR is a logical solution here, since such relations are born and develop within the network, so it makes sense to handle the related disputes internally, without transferring them to the real world..$^{45}$ As the business community already prefers ADR to litigation, there is no wonder they would consider resorting to some cyber alternatives of the same methods (and if none is available - invest in developing them from scratch). That is exactly what happened to eBay and TaoBao trade platforms, which were in desperate need of dispute resolution tools for their buyers and sellers. Eventually they came up with totally authentic solutions that are now cited as the most successful ODR examples. ${ }^{46}$

Online mechanisms of dispute resolution are especially welcome where the physical distance is the main obstacle for a proper hearing. In eCommerce it is highly likely that parties are not present within one jurisdiction, ${ }^{47}$ consequently it is easier to interact through an unbiased and neutral platform, such as the Internet. ${ }^{48}$ There is no need to travel anywhere, to look for a proper court or arbitrator to handle the case: the ODR provides an approachable solution for all. The other two things the business people may value are savings in money and time. We believe, it is obvious that online procedures take less time and do not require that much investment (including such obsolete things as travel expenses and costs for printing out the documents). Some expert estimations promise up to $30 \%$ savings in time and money when dealing with online mediation. ${ }^{49}$

All the things mentioned are especially inviting for the consumers (who, since recently, are also active online-buyers). Sometimes ODR may even be the only chance for them to get any relief. In eCommerce situations the other (business) party may be miles away, protected by foreign and unknown legislation, sometimes not even

42 P Noriega, C López, ‘Towards a Platform for Online Mediation' (2009) CEUR Workshop Proceedings 482.

43 A S Shetty, R R Pathrabe et al, Legal Issues in eCommerce < https://www.academia.edu/8148042/Legal_ Issues_in_E-Commerce> accessed 12 August 2019.

44 B de Vries, 'Book Review: Online Dispute Resolution: Challenges for Contemporary Justice' (2006) 15 Information \& Communication Technology Law 121.

45 D Sauliūnas, 'Alternatyvūs ginčų sprendimo būdai internetu (online ADR)' (2003) 41(33) Jurisprudencija 40.

46 Y Zhao, 'Rethinking the Limitations of Online Mediation' (2018) 11 American Journal of Mediation 164.

47 B L Beal, 'Online Mediation: Has Its Time Come?'(2000) 15(3) Ohio State Journal on Dispute Resolution 735 .

48 E Katsh, C Rule, 'What We Know and Need to Know About Online Dispute Resolution' < https://www. americanbar.org/content/dam/aba/images/office_president/katsh_rule_whitepaper.pdf> accessed 3 July 2019.

49 Online Mediation - MediasiMediasi <https://mediasi.nl/en/mediation/online-mediation> accessed 12 July 2019. 
possessing a physical address or due incorporation to be sued in a court of law. ${ }^{50}$ Moreover, a great number of consumer cases concern relatively small matters ( $\$ 5-$ $\$ 100$ in average). While being sensitive to consumers, these disputes are, beyond all doubt, unprepared to offline consideration, as the sole preliminary steps to be taken would consume much of the potential award. ${ }^{51}$ At the same time ODR helps cut down on travel expenses, production of documents, hiring representatives and so on. Online environment also smooths the inequalities between the business and the consumer in their negotiating power, especially in asynchronous communication. There is no more a situation where a company's representative comes with piles of documents and a well-prepared speech while the consumer is feeling lost and outnumbered. On the contrary, both parties exchange textual notes and have enough time to plan out their strategy. The reasons mentioned here have already been embraced by the European Union, which primarily targets consumer disputes in its recent $\mathrm{ADR} / \mathrm{ODR}$ instruments. ${ }^{52}$

Thus, parties involved in commercial activities are the primary beneficiaries of online mediation services, while time and money are the two things driving the parties to online mediation providers. However, these are not the only (and in many cases - the primary) reasons to move things online. One may think of other situations, in which a virtual mediator would better suit a person's needs than his offline colleague. Here, we may name situations where it is physically difficult for a party to attend live sessions (due to illness or permanent disability). ${ }^{53}$ Internet connections, on the other hand, are more approachable and really capable to evade most of the difficulties.

In yet other situations a physical meeting might be possible though highly undesirable as is the case with various kinds of matrimonial and family disputes, especially those involving interpersonal violence or abuse. ${ }^{54}$ Here joint mediation is not the wisest solution due to concerns of fear and intimidation a party may have, as well as a coercive pattern of control that may exist in a mediation setting. For such reasons it was generally supposed that cases like these are not suitable for mediation at all. ${ }^{55}$ However, the chance of the parties to work out the dispute themselves and to find a peaceful solution should not be neglected, especially when the fate of common children or the well-being of a victimized party are among the subject matters. ${ }^{56}$ For them, online mediation gives a chance to participate in a dispute resolution procedure without fear of meeting the other party. They may do it from a comfortable place with their friends being near. Whereas it is true that negative emotions come from direct face-to-face contact, maybe this is exactly what should be evaded (and not mediation as such). As was said, online mediation gives a possibility to interact in textual form, thus it is definitely a promising solution for former spouses. It must be added that family mediation is extremely helpful

50 N Ebner, 'E-Mediation' in M S Abdel Wahab, E Katsh \& D Rainey (eds) Online Dispute Resolution: Theory and Practice (Eleven International Publishing 2012) 362.

51 C Rule, 'Designing a Global Online Dispute Resolution System: Lessons Learnt from eBay' (2017) 13 University of St. Thomas Law Journal 356.

52 See Directive 2013/11/EU of the European Parliament and of the Council of 21 May 2013 on alternative disputes and amending Regulation (EC) No 2006/2004 and Directive 2009/22/EC (Directive on consumer ADR), OJ L 165, 18.06.2013; Regulation (EU) 524/2013 of the European Parliament and of the Council of 21 May 2013 on online dispute resolution for consumer disputes and amending Regulation (EC) No 2006/2004 and Directive 2009/22/EC (Regulation on consumer ODR), OJ L 165/1, 18.06.2013.

53 E M Lombardi (n 13) 541.

54 F S Rossi, A Holtzworth-Munroe (n 34) 399-400.

55 F Rossi, A Holtzworth-Munroe (n 34) 391.

56 A Kuhl, 'Family Law Online: The Impact of the Internet' (2008) 21(1) Journal of the American Academy of Matrimonial Lawyers 239-42. 
in countries with a mobile population. After a breakup, the spouses may settle in a new location and for that reason it is not their reluctance to meet face-to-face, but rather the impossibility of this due to new work and social commitments that presents a problem. They may, however, easily find some free time to conduct online meetings, or just to exchange messages in an asynchronous procedure.

With many other cases (employment, intellectual property, corporate and partnership, etc.) it becomes a general truth that the parties are annoyed or unwilling to see each other in person, while still opting for an amicable solution of their dispute. In that way, relations between former employer and employee may be strained, they may no longer be present in the same area, so online mediation gives them a proper place to voice their views and settle disagreements.

Online mediation has some perspectives in educational (both school and college) environments, conflicts between public authorities and citizens/businesses (e.g. parking, granting licenses, land rental, payment of taxes and much more). ${ }^{57}$ Potentially, this form of dispute resolution may embrace all areas of human activity that are not exclusively resolved for the courts (constitutional, administrative, most criminal cases). ${ }^{58}$ At the same time, even in public conflicts mediation may appear as a supporting tool, and thus its online form is also quite welcome.

\section{POSITIVE FEATURES}

Classic advantages of mediation are well-known and have been repeated many times. ${ }^{59}$ These are: confidentiality of the procedure, time and cost savings, control of the parties over the process, flexibility and predictability and some others. ${ }^{60}$ Many of them have already been discussed in this paper. What is important about mediation is its flexible nature, permitting those participating to feel more comfortable and cooperative. A great deal depends on the mediator, who is a necessary figure in the process: we presume that where parties resort to mediation, their previous efforts to reach peaceful solutions through bilateral negotiations have failed, thus a third neutral person is needed to maintain a balance and give each party a chance to express herself. ${ }^{61}$

It was mentioned that online mediation is cheaper for the parties, but it also provides for some savings to the neutral intermediary. The latter does not have to rent an office and bear associated costs, ${ }^{62}$ however, in some cases his expenses for professional software licenses, data protection and cloud storage may even exceed those of offline mediators. Nevertheless, what is definitely expanding is the possibility of a mediator to practice around the globe. With a laptop as his main tool there are no geographic limits to offer the corresponding services. ${ }^{63}$ The market is quite competitive, yet a solid professional will always be able to find his niche. From the participant's perspective such situation is

57 F Petrauskas, E Kybartiené, 'Online Dispute Resolution in Consumer Disputes' (2011) 18(3) Jurisprudence 924.

58 S S Raines, 'Can Online Mediation Be Transformative? Tales from the Front' (2005) 22(4) Conflict Resolution Quarterly 449.

59 S N Exon, 'The Next Generation of Online Dispute Resolution: the Significance of Holography to Enhance and Transform Dispute Resolution' (2010) 12 Cardozo Journal of Conflict Resolution 24-6.

60 K Taylor, Technological Advancements Help Resolve Disputes <https:/www.mediate.com/articles/ TaylorKbl20151218.cfm> accessed 5 August 2019.

61 S N Exon (n 59) 24.

62 D Lavi (n 36) 289.

63 G Ross, ODR's Role in In-Person Mediation and Other 'Must Know' Takeaways About ODR <https:// www.mediate.com/articles/RossG2.cfm> accessed 2 July 2019. 
also quite favorable, as his home region may offer services of poor quality or none at all. The Internet, however, permits to find a specialist on the other side of the world, and the only big obstacle is, seemingly, the language barrier.

Many inherent advantages are attributed to textual communication (the main medium of online mediation). This way, the parties have more time to think about their answers instead of hurrying up and saying aloud many undesired things. Their messages are more likely to be a thorough and logical proposal than the one coming from a face-toface hearing limited in time.

To some, the significant benefit of text-based mediation is a possibility to eliminate bias based on race, age, gender or disability. ${ }^{64}$ Neither the mediator, nor the parties normally know, who is behind the screen, thus they may focus on essential points of the dispute rather than a specific characteristic of another participant triggering some form of prejudice in their mind. Since there are no 'faces', but just 'accounts' or 'user profiles', all the subjects in the process may genuinely feel themselves equal.

Last but not least, online mediation may be essential in cases where everything else fails. Some of the relations existing on the net are not properly regulated by the law with none efficient remedies offered in case of violation or abuse. To this category we may include disputes between online gamers over some in-game artifacts, not being considered as 'real property' in classic terms, yet quite valuable and important for the relevant community; and also inter-personal conflicts in social media, which can only be comprehended by someone, who is also a part of the same community and knows its hierarchy of values and codes of conduct. As is clearly seen, online arbitration and negotiation are quite likely to fail in both mentioned cases, thus a procedure involving a charismatic and persuasive intermediary needs to be put in place.

\section{5. (UN)OBVIOUS DRAWBACKS}

In traditional mediation the key issue is that of trust. This broad term embraces trust between the participants, trust of each party to the mediator and, finally, confidence in the procedure itself. With online mediation we can safely add trust to technology behind the process to the list. Without proper trust established the parties will not be willing to act in a co-operative manner, share their thoughts and feelings, to exchange sensitive data and so on. Ultimately, they may even opt out of the procedure, preferring to bring their dispute further to court, the powers of which are undoubted.

Unfortunately, the Internet, in general, has developed into an environment fraught with distrust. ${ }^{65}$ Reasons for that are different: the big number of frauds, impossibility to get enough information about another party, let alone a general fear of the unknown. In fact, we have to build up this trust from scratch when moving online.

Despite some examples of effective synchronous text-based communications (such as chats or web-messengers), offered by some major ODR platforms (such as ODRWorld or RisolviOnline), the standard in the industry is still asynchronous communication. ${ }^{66}$ This comes at odds with the very idea of traditional mediation, i.e. that it has to be

64 P Young, Online Mediation: Its Uses And Limitations <https://www.mediate.com/articles/young4.cfm> accessed 22 July 2019.

$65 \quad$ N Ebner (n 50) 369.

66 Id 370. 
face-to-face, not screen-to-screen. ${ }^{67}$ For some professionals, the concept of indirect mediation presents a complete nonsense. ${ }^{68}$

These negative concerns have their grounds: indeed, such process as mediation requires a deep personal connection. With text-based communication you never know who is behind the screen and what are their true intentions. If you are unfamiliar with another side, it is extremely difficult to 'humanize' it, that is, to start thinking of that other party as the same human being with all the usual feelings and propensity to errors. Text typed online and delivered by the machine cannot help to catch person's emotions, feelings and desires. What one can see is just a bit of words written by 'someone'. Such form of communication is much more stale, emotionless and straight. Where interaction is implemented by means of email or messenger notes, another important feature comes out. People normally try to sound more professional, solid and reliable while composing their letters. When the use of email is at stake people normally resort to standard templates and set phrases, which include formal words and expressions devoid of any practical meaning and thus useless for the other party and mediator to identify their true intentions.

It is also quite easy to understand the promise of a letter in a wrong way. A joke included by the party to defuse the situation may be considered to be a mockery, and a reasonable proposal - as a gimmick. It must be observed that this argument does not work in case of family or matrimonial mediation where the parties are already familiar with each other and are less likely to misinterpret words and intentions of another participant. Some also argue that in eCommerce disputes the textual nature of mediation does not pose serious problems, as they are often non-personal (but rather, monetary). Consequently, there is no need for the parties to go deep into building up a picture of each other's character. Their initial decision to resort to online mediation shows their intention to negotiate and to peacefully settle the problem, going on with their business. Although somewhat reasonable, this argument misses a mediator's role in the procedure. He is also quite limited by the chosen medium and may have a hard time getting information about the parties. ${ }^{69}$

An interesting observation also showed a stronger prevalence of aggressiveness in online communication. People online are more likely to insult and offend each other, as well as to say words that would never sound in face-to-face communication..$^{70}$ Such inflammatory comments and ad hominem attacks are quite unusual for traditional mediation where the parties all appear in the same room. ${ }^{71}$ Decency, tact, education, in the end, fear of rebuff all act as psychological barriers to aggressive attacks. With distant contacts, however, parties start feeling that they can get away with anything, as they can always quit the discussion and, at the worst, turn off the computer. Although in online mediation parties are aware their partner is a real person, their online habits of treating others may still come out. ${ }^{72}$

Textual communication also shows its drawbacks when speaking about the ability to logically and clearly present one's thoughts and some literacy. As mentioned previously, web-based media make communication less biased, as we do not see who the parties are in reality. Thus, their rhetoric skills and ability to win the crowd do not bring any

67 M Albornoz, N González, 'Feasibility Analysis of Online Dispute Resolution in developing Countries' (2012) 44 University of Miami Inter-American Law Review 39.

68 A M Braeutigam (n 23) 276.

69 A Schmitz, 'There's An “App” for That: Developing Online Dispute Resolution to Empower Economic Development' (2018) 32 Notre Dame Journal of Law Ethics \& Public Policy 2.

70 N Kravec, 'Dogmas of Online Dispute Resolution' (2006) 38 University of Toledo Law Research 130.

71 P Young (n 64).

72 D Lavi (n 36) 297. 
additional points. However, in online mediation people do not become fully equal, as there is still a divide based on their literacy. A mediator might feel more respect towards a person with solid writing skills rather than the one making constant mistakes and being unable to convey his idea. ${ }^{73}$

Another thing that calls for rethinking is the idea of faster procedures in online surroundings. It was said that since parties do not need to travel anywhere, the mediation procedure will require less time. At the same time, since in most cases we talk about the textual medium, things may be quite the opposite. ${ }^{74}$ It takes time for parties to read a message and craft their reply. Sometimes, we may face delays just because of the inability of the participant to keep with the pace of the procedure. The main reason is the lack of temporal discipline that in normal mediation is remedied by the mediator and the general atmosphere in his room. In fact, a professional offline mediator may be able to resolve the dispute in one day. His online colleague might want to beat that record, yet some aspects of the process (such, as the time it takes for a party to reply) go beyond his control.

This lack of effective control is another weakness of online mediation. It is not possible to use uninterrupted mediation, as well as to impose a 'cooling off' period on the parties (especially where the communication takes place via email or another medium beyond mediator's control. In such situation a good mediator knows in advance that it is better to stop and let the parties some time to calm down. During this period they do not meet in person before the mediator and normally do not talk to each other in any other way. As mentioned by Raines, she was unable to impose a cooling off period on the parties in an online mediation setting where email was the main means of communication. ${ }^{75}$ Since the means of communication was outside of her control, there was nothing she could do to ensure compliance. Eventually, the parties continued exchanging offensive letters insulting each other and escalating the conflict, without mediator even knowing of what is going on.

A situation where the mediator does not see messages prior to them being sent (or, in the worst case, not seeing them at all) makes him totally blind and unable to track the progress in the dispute. In fact, at some stage he may be totally and implicitly removed by the parties from the loop essentially turning the whole process into another form of dispute resolution - negotiation. The only thing that can motivate parties to return to mediation is their advance payment for the whole procedure.

Quite tense is also the situation with private data of the participants and confidentiality of the whole procedure. As known, traditional mediation is a highly confidential procedure with all information revealed during the sessions kept in secret by the parties and mediator. The latter, moreover, is placed under an obligation to keep everything in secret, and many national laws even presume that they cannot be compelled to give evidence regarding information arising out of or in connection with a mediation process. ${ }^{76}$ Information is only shared in oral form, and never leaves the mediation room. With online mediation the things are, however, becoming more complicated. Since we talk about online platforms and special applications, we presume that textual data shared by the parties are stored somewhere on the web server. In other words, there is a digital trail left after each session.

73 B L Mann, 'Smoothing Some Wrinkles in Online Dispute Resolution' (2009) 17 International Journal of Law and Information Technology 110.

74 See J C Betancourt, E Zlatanska (n 17) 258.

75 S S Raines (n 58) 448-9.

76 EU Mediation Directive (n 10), Art. 7; Lietuvos Respublikos mediacijos įstatymas, Valstybès žinios, 2008-07-31, Nr. 87-3462, Art. 17. 
This brings us once again to the question of trust - what is the chance that the sensitive information kept on the server would not be transmitted, leaked or otherwise made available to the third party? One may think of deliberate hacker attacks or simple provider's negligence in keeping the data well-protected. Surely, most ODR services provide in their terms and conditions that the confidentiality of information is guaranteed and all the materials are duly encrypted, ${ }^{77}$ however, these are only standard promises and their sincerity is in question. In fact, most commercial organizations cannot guarantee a decent level of privacy and data protection. Even such powerful corporations as Facebook or Microsoft get hacked or leak private information of their users, what to say of small private ODR providers with limited cyber-security budgets?

The problem is not only in third parties' unauthorized access to the data, but also in the potentially unfair conduct of the other party to the dispute. Technology permits recording. Even when the mediator ensures that the system makes no record of the communications, it is still possible that one of the parties uses a third-party application to take screen shots or record audio- and video conversations. The information retrieved might be later used to blackmail the opponent or to cause damage to his reputation by publicly distributing it, while it is almost impossible to somehow prevent such illegal behavior. In the end, this may lead to two possible situations: parties will either fear to disclose sensitive information, which will in turn harm building trustful relations between them, or they will totally ignore OM for the fears named above, instead opting for traditional mediation or other dispute resolution techniques.

For all forms of ODR the technological gap has always been considered a significant issue. In order to participate in online mediation you have to be familiar with modern technology. Consequently, a new form of social inequality comes to the stage: that of computer literacy. Advanced users get priority, while ordinary citizens (especially those living in rural areas) lag behind without any fault on their side. This problem of the digital divide might seem to be far-fetched and no longer valid in the year $2019 .{ }^{78}$ Firstly, the main target audience for online mediation becomes the younger generation, which actively uses ICT for both private and professional needs and finds no difficulties in getting familiar with some new application. Secondly, it is incorrect to presume that access to the internet is limited. In fact, one does not need a laptop to connect to the network, as modern tablets, smartphones, e-readers provide for that. In any case, online mediation websites can be accessed through public places (libraries, schools, universities and the like). Thirdly, it must not be forgotten that mediation is a voluntary process, thus a party feeling uncomfortable in the online environment in any case can refuse to participate from the start.

In their turn, proponents of the digital divide problem insist on drawing the line between simple web-browsing (which is, indeed, open to any modern person) and using the Internet for professional (job- or business-related purposes). Only the latter form presupposes the knowledge of specific means of online dispute resolution and frequent resort to them in practice. However, an ordinary consumer may know even less about online mediation than he does of its offline counterpart.

\section{SOLUTIONS AND POTENTIAL FOR IMPROVEMENT}

In this section, we will try to see how different problems named above may be solved. For many scholars the most significant drawback of online mediation is its text-based nature, thus it may be overcome as technology advances and high-speed Internet connections

$77 \quad$ D Lavi (n 36) 296.

78 P Cortés, Online Dispute Resolution for Consumers in the European Union (Routledge 2010) 58.

TEREKHOV V. ONLINE MEDIATION: A GAME CHANGER... 
will allow for videoconferencing. As suggested by Beal ${ }^{79}$ and Goodman, ${ }^{80}$ only with the dissemination and promotion of videoconferencing will we be able to witness the true success of online mediation? In their view, the current trends of primarily text-based procedure present a temporary juncture that is likely to change with the evolution of technology and it's becoming more available to wider audiences.

However, we find it difficult to agree with the authors. Videoconferencing (as it is available nowadays) is not nearly as efficient as oral communication (which it is called upon to replace): in most situations it is only possible to see a person's head on the screen, thus leaving participants without other important cues (gestures, poses, handshaking, etc.). Audiovisual channels used for transmission are also far from perfect. They are susceptible to disruption and deterioration; a connection may be lost or its quality may be far from perfect. Moreover, it shall not be forgotten that some people opt for online means of resolution precisely for a chance to stay 'anonymous', ${ }^{81}$ if only that means a chance to withdraw from showing one's real face. For a younger generation text-based communication largely presents the 'new normal' as much of their interaction with friends, family and colleagues takes place via messengers. ${ }^{82}$

To conclude here, text-based procedures are not just interim measures designed for a transitional period (until videoconferencing becomes widespread). Instead, they are rather here once and for all and we have to embrace their principal role in internet dispute resolution.

It does not mean we are turning a blind eye to the problems of textual communication. Instead, we propose to solve them through changing the role and some functions of the mediator in such proceedings. After all, it is his proficiency and determination that drive the whole process. First of all, this person will need to dig deeper into the texts they are working with, learn to read between the lines. It may happen that quite often the mediator will have to ask leading questions ('did you mean X by saying Y?', 'did I get it correctly that you find Z's proposal unacceptable?') or suggest reformulating some of the ambiguous sentences. Some neutrals get permission at the outset of the mediation to filter certain types of comments by returning them to the sender for redrafting. Only afterwards they are delivered to another side of the dispute. Such a practice seems essential in overcoming misunderstanding.

As for the poor means of expression available in textual mediation, one needs to remember the following. The whole procedure bears a remarkably non-formal nature. In online interaction it is obvious more than ever. The target audience for online mediation is internet people, already bearing this kind of informal culture. They bring it together with them to their disputes, turning it into a universal standard. It may well happen that the use of emoticons, capital letters (the online equivalent of shouting) and specific slang may become widespread in online communication and even be partially adopted by the mediators. All these techniques will definitely help compensate for the lack of cues available during direct meetings. In fact, although yet to be proven by research, the modern generation may feel more comfortable with available textual means than

\footnotetext{
$79 \quad$ B L Beal (n 47) 735.

80 J F Goodman, The Pros and Cons of Online Dispute Resolution: An Assessment of Cyber-Mediation Websites < https://scholarship.law.duke.edu/cgi/viewcontent.cgi?article=1073\&context=dltr $>$ accessed 2 July 2019.

81 'Anonymous' - a name of decentralized international hacktivist group as well as web-movement, the central idea of which is a human right to full anonymity for internet users in all forms of their interactions.

82 A Kuhl, 'Family Law Online: The Impact of the Internet' (2008) 21(1) Journal of the American Academy of Matrimonial Lawyers 226.
} 
with oral communication with an unknown person in unfamiliar surroundings (like mediator's cabinet) ${ }^{83}$ In this way, text-based communication is not inferior to face-toface meetings, but rather presents a different mechanism of doing the same thing negotiating over a dispute with an intention to reach a peaceful settlement.

In order to deal with aggressive speech, mediators may resort to an already mentioned tactics of pre-moderation of the messages that parties intend to deliver each other. Modern software may also do quite a lot to paraphrase sentences and delete offensive words. However, in order to ensure better effect, it makes sense that a human mediator as a third neutral party reads through the lines and shows parties which parts of their postings may sound odd or lead to unwanted results.

A mediator may also reframe certain words or sentences in order for them to sound more natural, clear and close to what the party had in mind. There is, however, another problem: people may oppose such censorship, which does not have direct equivalents in traditional mediation. Where in normal mediation you can freely express your thoughts, here you have to deal with a neutral, who comments on your phrases and may misinterpret them much like the other party. No definite solution to this concern may be presented now. It seems that much depends on the mediator's ability to win the party's trust and show he is there to help, which in turn presupposes his need to intervene within an inter-party communication to break the tension and ensure the cooperative atmosphere. It is also important to recommend rather than command. A kind note on the incompatibility of the wording shall always be preferred to strict rejection of party's text. Instead of criticizing a party for choosing wrong words and tone, it is always better to point out which additional things could be mentioned and what kind of style would look more attractive.

Returning to the problem of the digital divide, it must be observed that it shall now fall within the official duties of modern mediators to clarify and make obvious for the parties what is going to happen on the whole course of online mediation process. At the same time mediator needs not to be regarded as an IT consultant, thus questions on how to send messages, upload/download files, join and leave virtual meeting rooms must be addressed to specialized technical staff. For practicing online mediation it requires hiring some support specialists trained in those questions and paying them on a regular basis.

Technology plays a great role in online mediation as well. It can allow for direct talks between a mediator and each of the parties, remind of important deadlines, propose words and phrases to be included in response to the other party and ultimately - even draft a preliminary settlement agreement. The software tools need neither be demonized nor worshipped. They simply fulfil their tasks and may improve with time (in case enough feedback is received from previous participants).

\section{CONCLUDING REMARKS}

It is obvious that modern society lives on the Internet. According to some statistical data, time spent online 'equates to more than 100 days [... ] every year for every Internet user. ${ }^{34}$ Dispute resolution techniques are a necessary component of this web-based community, and these have to be fast, easy, costless, effective and efficient. ${ }^{85}$ Mediation

$83 \quad$ E M Lombardi (n 13) 541-2.

84 M Hughes, Study Shows We're Spending an Insane Amount of Time Online < https://thenextweb.com/ tech/2019/01/31/study-shows-were-spending-an-insane-amount-of-time-online > accessed 2 July 2019. 
was not designed for the Internet when it originally appeared, yet it fits perfectly within this environment. It is flexible and informal, much like other things in the World Wide Web. It does not present a binding form of dispute resolution, but that is precisely what allows it to survive jurisdictional and political pressure. In other words, we are sure that online mediation will survive as such, however, its exact perspectives are far from clear.

The most important concern is the ability of mediators to rearrange their tactics and learn new tricks ensuring the quality and success of the procedure. For those who believe online mediation to be just involvement of several ICT tools there may be a problem to adapt to new realities. On the other hand, those ready to evolve, learn and sometimes test new techniques, will be able to benefit from getting a share of the new, huge and innovative market.

Online mediation is still a developing area, without firm rules and boundaries. Only time will tell whether it turns into a trustworthy and authoritative form of dispute resolution, or occupies a marginal place among other ODR methods. 